\title{
Wuxing Theory Reflecting in English as Foreign Language Teaching
}

\author{
Bulei Zuo \\ School of Foreign Languages, Soochow University, China
}

\begin{abstract}
The theory of five elements, the core of Chinese ancient philosophical framework is the holistic view of thinking as the simplest concept in Chinese traditional culture. Researches on this theory applied in linguistic study are still blank. This paper attempts, from the yin-yang perspective of five elements generated in foreign language teaching practice, to explain and demonstrate the correspondent relationship among five teaching elements in EFL, to explain the phenomenon of internal changing and reinforcing each other, over-restricting and point out that the construction of "five-element teaching theory", a mode of thinking, is beneficial for promoting effective Foreign language teaching.
\end{abstract}

Index Terms-Wuxing, holistic view of thinking, five-element teaching theory

\section{INTRODUCTION}

\section{A. Wuxing}

Wuxing, sometimes translated as five elements or five phases, is primarily an ancient perceptive device in ancient Chinese philosophy. The Five-Element Theory is the fundamental code of systems thinking of ancient Chinese, which, based on the principle of analogy, is applied to classify all phenomena of the world into five categories according to the properties and characteristics of WOOD, FIRE, EARTH, METAL and WATER. The interrelationship among them can be used to explain the movement and transformation among all material things (Zhanwen Liu, 2009). Being different from Western conceptual logic thinking, the five-element thinking brings about a whole system thinking model, a harmonious manner, which has been guiding Chinese people to percept and interpret various phenomena existing in wonderful nature, human body, empirical world and teaching field.

\section{B. Wuxing Teaching (Five Teaching Elements)}

Creating an effective classroom for Chinese English learners is challenging and time consuming for English teachers, but it is definitely worth the effort. As the classroom is composed of a variety of micro ecological environment factors, also called teaching and learning elements, which have to be maintained in dynamic balance when learners are participating in various activities (Harmer, 2000). In language classroom, there are at least five elements which should be taken into consideration when we are required to solve some problems on second language learning and language teaching: learners (learners' interest, attitude and motivation), learning strategies (approach, method), teacher (passion, professional development), teaching method (techniques, approaches) and teaching material (textbook, information source). It is worthwhile to explore the issues of EFLT (English as foreign language teaching) from the perspective of Wuxing. The system of five elements was used to describe interactions and relationships between phenomena. As a device, it was employed in many fields of early Chinese thought, like martial arts, cosmology, TCM(Traditional Chinese Medicine), music, military strategy, and language education (Wang Ningchuan and Zou Yuze, 2011). The five elements are infinitely linked, consuming and influencing each other.

\section{Why Five Elements, Not Six or More}

Wuxing, the Five Elements is originated from Chinese traditional culture. Why are there five, not six or seven elements? One reason is that the number "Five" is in the middle way. The "middle way", belongs to the category of ancient Greek philosophy, Pythagoras School thinks the existing things being in "balance between each oppsite one to a proper extent". Platon started to transplant this concept of equilibrium into ethics, and Aristotle established a connection between the doctrine of the mean and the control of action and thought that everything has the middle way, such as the number " 5 " is in the middle of the numbers from one to ten.

The other reason lies in the fact that in Chinese traditional culture, Yin and Yang played most important part in holistic way of thinking. The Chinese are accustomed to use Yin and Yang, the two elements to represent the opposite properties in the universe, such as masculine and feminine, lower and upper, cold and hot, slow and fast, still and moving, etc. as an ancient Chinese philosophy that is represented by the Tai-Ji symbol, a circle with black and white shapes existing in equal proportion inside its outline, meaning the bright and dark sides of an object in Chinese. In EFTL (English as a foreign language teaching and learning), learners and learning strategy pertain to Yang and Yin; teacher (passion, professional development) and teaching method (techniques, approaches) also pertain to Yang and Yin. Textbook (material, context, 
task) and language atmosphere (something textbooks provide) have Yang and Yin attributes. However, language atmosphere is the product of integrated effect and combination made by learners and teachers as well as their strategies and methods based on the comprehension of material, information source. Language atmosphere, as the sixth element, invisible but most important one, refers to outside margin of Five-Element Thinking Model. For the sake of simplicity and convenience, we Chinese employ the five-element modelto illustrate their inner ralationship, easy to read and easy to recognize. Most importantly, it is consistent with Wuxing Diagram of TCM. In TCM, we Chinese understand that there are natural laws and energy forces implied in the elements, which can be used to help diagnose and treat the cause of the problem, and calm our mood generated in spirit and body, unblock meridian channels and allow our energy to flow properly (Wang Aiping, 2012). So does EFT (English as Foreign Teaching). It is impossible to have full healthy educational situation if there are any mental, physical or emotional problems blocking our thinking and active practice. The five teaching elements need to be rebalanced by breaking through the key links of "meridian or meridians" (rationales, concepts, strategies, methods and designs), leading learners and teachers back into the full healthy teaching and learning situation.

The reasons seem to be subjective, but do matter objectively. Actually there are variety of elements affecting the EFL and EFT. The author just integrates those into the five, abstract, not concrete. Additionally, Wuxing Teaching Model, the Five Teaching Element Model shows, in broad sense, that there are interrelationships among any factors or elements which exist here and there, even though there is always at least one dominant element which plays an important roloe in affecting language output. When we explore any language theories and practice them, we do need to stop to think before action.

EFT through the perspective of Five-element Theory, does interest students who as well as teachers can understand the relationship amnong such five elements as learners, learning strategies, teacher, teaching methods and textbook. This article will suggest a motivating way to teach English with perceptions of relationship of "Five teaching elements", and will attempt to explore the possibility and feasibility of applying the five elements theory into foreign language teaching, in term of holistic way of thinking.

\section{Classification And Attribute}

By employing the method of "classifying by analogy", the ancients established extensive connections among five categories which have similar properties and appearance of WOOD, FIRE, EARTH, METAL and WATER existing in a system. Luckily, there are five corresponding five elements in EFL: learners, learning strategies, teacher, teaching methods, teaching materials (textbooks) (Xia, Songlin, 1989). It is necessary to clarify attributes of these five elements before mapping them onto language teaching system. Actually, Chinese systematic way of thinking focuses on dynamic functions and explicit effects of the five elements, which can be easily understood in terms of the basic seasonal cycles of nature (Liu, 1990). Over long-term observations of the properties of the Five Elements, the intelligent Chinese gradually formulated a more abstract conception with their own characteristics, implied with wide range of application as follows.

\section{A. Learners $(L)$ as WOOD}

Wood means creative energy. Element WOOD is characterized by uprising, thriving, flourishing, generating, stretching, smoothing, literally springing forth of growth after long period of hibernation. WOOD, especially in spring, like the start of new life cycle, is associated with vigor, youth, and growth. It in energy is creative and expands up forward freely. In classroom activity, language learners, who have positive cognition, intrinsic motivation for meaningful learning, self-confidence, strategic investment of time, effort and attention (H. Douglas Brown, 1994), could be analogized as WOOD, allowing to be carved, straightened, a metaphorical expression, as they govern information flow, and are eager to grow and develop independently, and they, full of implicit creative energy, has the ability to bend, to extend, to break free, to rise and to flourish, and has guided the unformed energy of WATER (Wisdom, mentioned below) in language learning to an applicable and viable direction targeting at marketable skills and nice competence of a certain foreign language, just as the green plants growing upward from the ground. In Chinese traditional culture, learners are addressed as "one-hundred-year wood man", indicating the hard work to educate a successful talent.

\section{B. Learning Strategies (LS) as FIRE}

Fire means the one of enthusiasm. Just as spring goes naturally into summer, temperature rises and everything tends to be flourishing, changing, ascending with the warmth and vitality from the sun, symbolizing the flourishing phases of life cycle. So things that are characteristic of heat belong to FIRE, blazing upwards. For educational purposes, the effective learning strategies, the right learning methods and techniques, and right amount of practice (hear after as LS), which learners manage to find to make things done, are like a torch, giving out light, flaming and ascending. Chosen by the intelligent learners LS seems to become FIRE that promotes their understanding of what they have learned with new ideas. Learning strategies function as satisfying students' needs, matching their desire, like the living beings flourishing under the warm and stable glow of teacher's encouragement.

Jeremy Harmer.(2000) thinks that a successful learner should have the willingness to listen, to experiment, to ask questions and to think about how to learn, which implies the essence of learning strategies; H. Douglas Brown (1994) states that a classroom learner should have strong language ego(a second language identity), and risk-taking to ask 
questions. Asking question is a remarkable symbol of holding a certain learning strategies so that the specific learning strategies like autonomous learning, collaborative learning, group learning etc., could become possible. Confucius once said, classroom teachers need to continue to ignite the fire-like enthusiasm of the learners, encourage for extensive learning, intensive questioning, deliberating, and discerning in constructive way. The "fire" attribute is given to the cultural connotation of Learning strategies, which is YIN, and learner is YANG, so the integration of Yin and Yang, creates a whole and healthy learner.

\section{Teacher (T) as EARTH}

Earth symbolizes fundamental basis for gardeners. Element of EARTH is the source of everything which represents the sowing, reaping, producing and engendering transformation. Autumn is the typical season when things form and sow, characterized by storage and accumulation for use in non-productive winter. Such life phase has reached maturity and harvest, implying fruit of previous efforts. Correspondingly, in EFL or ESL classroom, teachers symbolize the source of knowledge (H. Douglas Brown, 1994). Teachers who have strong sense of responsibility, love and patience can be categorized as Earth. Teachers are just like power station, functioning as activating learners' interest in learning target language, and as a farm for learners to plant and harvest, receive and bear, even to nourish. When the energy of EARTH transforms into the reaping stage of energy in golden season, METAL appears. In other words, if language teachers are well-qualified and capable enough, textbooks and productive learning materials can be written and used properly. In Chinese culture, teacher is regarded as someone owning heavily virtuous qualities and carrying heavy objects on earth, as the Classic of Analects mentioned. In this research, teacher refers to the professional person who has a good command of English and more elements of professional competence (Anna Craft, 2002).

\section{Textbooks (TB) as METAL}

Metal means reaping energy. The fall, a harvest season, is featured by convergence. As METAL is characterized by "malleability", allowing to be molded, which derives from the fuction of METAL to conform to external forces despite its strength. In EFL, textbooks (TB), including learning material, any video and audio information sources, as well as right learning atmosphere, the teaching and learning contexts learning environment like library, classroom and dormitory could be categorized as METAL, as it is the working force of change, a source of information, wisdom and intelligence shared, analyzed and communicated. Its core function is to work as a tool, a media, and interactive platform for teacher and learners to convey literal message and improve language competence.

\section{E. Teaching Methods (TM) as WATER}

The element WATER symbolizes the winter-like wisdom ini Chinese culture. Water possesses the characteristic of being wetting and descending, moistening downward and being condensed and conserved. The reserved harvest saved in METAL phase is for the renewal growth in WOOD season. It will be pregnant with potential energy for new life, which can be abstracted as the characteristics of WATER: moistening, moving downward, cooling, and concealing. Similarly, the teaching methods (hereafter as TM), including practical approaches, the skills of organizing variety of classroom activities acts as WATER in the five-element model. For students in school, teaching approaches function as intangible asset that can not realize its value until it is highly effective, tangible, close to learners' daily life or previous experience. Like the attribute of WATER, teaching approaches should be flexible, changeable and reachable. Take the students' feeling awkward when speaking English for example, the solution is to create a very warm, inviting, and risk taking atmosphere in the classroom. which is the function the teaching methods works serves. Another example to illustrate the function of teaching methods is that a language teacher should instill in his students a number of skills that are more directly related to the students' psychological attitude toward new languages than to their direct knowledge about the language they are learning (Adrian Palmer,1970). In language history, such teaching methods as the Grammar Translation Method, the Direct Method, the Audio lingual Method function well in a certain period (Shih-Chuan Chang, 2011). However, in this research, teaching methods include vaguely approach, techniques, even though Edward Anthony (1963), Richard, Jack and Theodore Rodger (1986) defined those terms clearly.

\section{Mechanism of Reflecting the WuXING Teaching TheORY}

\section{A. Wuxing Generating Relationship}

\section{Generating Circle in Wuxing}

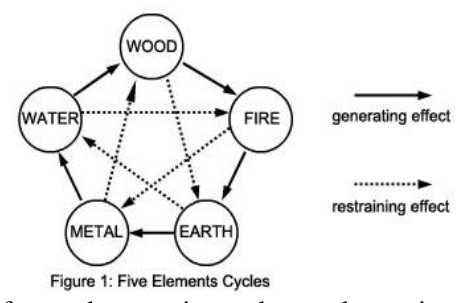

Figure 1 presents the relationships of mutual generation and mutual restraint among the Five Elements graphically. 
There are two basic systemic and regulatory ways associated with Five Elements: generation and restraint. Generation and restraint are a combined fundamental principle which is applied to encompass and explain the relationships between things, and their development and changes. The concept of generation contains the ideas of production, stimulation and augmentation with a cyclic sequence of generation like this: WOOD feeds FIRE, FIRE creates EARTH, EARTH generates METAL, METAL carries WATER (as in a bucket or tap, or water condenses on metal), and WATER nourishes WOOD. There are two aspects to the relationship of generation for each element, those of "being generated" and of "generating." The former is the "child" of the generating Element and the later is the "mother" of the generated Element Hence, the generation relationship is also known as the "mother-child" relationship. Take WOOD for example. WOOD generates FIRE; thus FIRE is the child of WOOD, and WOOD is the mother of FIRE. The other four Elements follow this illustration (Zhanwen Liu, 2009).

The above mentioned classification will be evidenced by interrelationship of mutual nourishing aspects in EFL system which demonstrates that there is also a relationship of opposition and unity between the internal environment of the classroom and its external surroundings, a relation of maintaining a dynamic balance. In classroom activity, L (learners)belongs to WOOD, mixed with LS (learning strategies belonging to FIRE). L governs LS, who has conflicting ideas and different needs for personal growth, interacting with the teacher that governs teaching approaches within a context the textbook supports. The context made by teacher and learners is based on teaching energy field, as well as learning energy field, the driving forces called "Qi", a dynamic language atmosphere. It is Qi that makes five teaching elements moving and changing.

2. Reflections of the Generating Circle in EFL $(\mathrm{L} \rightarrow \mathrm{LS} \rightarrow \mathrm{T} \rightarrow \mathrm{TB} \rightarrow \mathrm{TM} \rightarrow \mathrm{L})$

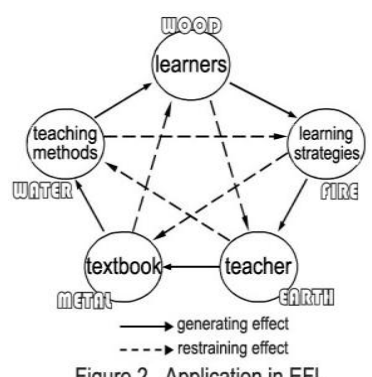

Figure 2 Application in EFL

L generating LS: Learning strategies are the actions that learners take in order to decode, process, store and retrieve language (Ernesto Macaro, 2008, p.109).

LS generating T(teacher): The necessary strategies competence needs to be trained by the teacher, so they can get that language into their mind in the give-and-take meaningful construction.(H. Douglas Brown,1994, pp.190-191).

$\mathrm{T}$ generating $\mathrm{TB}($ textbook): As to the relationship between teacher and textbook, a teacher should be skillful in handling the textbook. If the language, content and sequencing of the textbook are appropriate, the teacher will want to go ahead and use it. However, normally there is something wrong with the textbook, the teacher has to decide what to do next, like developing a kind of "pick and choose" approach to what is in front of them. According to Neville Grant, Neville Grant (1987) thinks that there are four options for teachers: omiting, replacing, adding and adapting. The textbook use as one of a teacher's main skills, like Earth bearing Metal. A very nice comment about teacher's role in choosing and using a textbook, is from J. Myron Atkin (1967) who compares teaching textbooks to the craft of making metals. For centuries and continuing today, says Atkin, skilled craftsmen have been making metals. They have learned to add a little of this substance and a little of that, then heat the batch for a certain length of time until it reaches a certain color, then let it cool at a certain rate (Atkin, 1967). The effectiveness of using textbook means the teacher generates appropriate teaching methods (TE nourishing TM).

TB generating TM(teaching methods): As for the relationship between textbook and teaching methods, H. Douglas Brown(1994:151) pointed it out that "textbooks" are one type of text, a book for use in an educational curriculum, with a wide variety of types or genres of linguistic forms: spoken or written. Among written texts, the range of possibilities extends from labels and forms and charts to essays and manuals and books, which are for use in supporting techniques in the classroom (H. Douglas Brown, 1994, p.151). There are dozens of resource books that are specifically designed to provide ideas for teachers, like role play, listening and speaking techniques, and activities for children. To sum up textbook generates teaching methods naturally.

TM generating L: Teaching methods focus on the techniques that accounts for learners' needs included in learner-centered instruction, which gives some control to the student like group work or strategy training (Mattarima, Karim and Hamdan, Abdul Rahim,2011), allows for learner creativity and innovation and enhances a learner's sense of competence and self-worth(H. Douglas Brown, 1994,p.80), that can generate more motivation (H. Douglas Brown, 1994, p.213). Another example of TM generating L is that as language teachers, they should constantly serve as models by illustrating appropriate language, demonstrating activities as well as giving instructions, and showing students how to complete assignments. In any classroom setting, teachers must show students what to mean and what to expect them to do, 
so that capable students can be produced. Getting students to do various kinds of homework like written exercises, composition, essay or study is the best way to encourage student autonomy (Jeremy Harmer,2000)

\section{B. Wuxing Restraining Relationship}

\section{Restraining Circle in Wuxing}

The concept of restraint contains the ideas of restriction, check and inhibition with a cyclic sequence of restraint as follows:

WOOD restrains EARTH, EARTH restrains WATER,WATER extinguishes FIRE, FIRE melts METAL, and METAL chops WOOD, among which two roles as to the relationship of restraint for each Element has to be mentioned, those of "being restrained" and of "restraining." The restraining Element is the "suppressor" and the restrained Element is the "suppressed." Hence, the relationship of restraint is also known as the "suppressor-suppressed" relationship. Again, take WOOD for an example. METAL is the suppressor of WOOD, and WOOD is the suppressed of METAL. In the theory of the Five Elements, each Element has a direct relationship with all the other Elements. For example, EARTH is the mother of METAL and the child of FIRE, and at the same time it is the suppressor of WATER and the suppressed of WOOD.

It should be noted that the two relationships of generation and of restraint are inseparable. Without generation organization cannot be born and cannot develop. Without restraint, organization can grow without limit and cause harm. It is necessary to keep both generation and restraint in harmonious relationships within an organization, and to assure their normal development and change. The essence of the theory of the Five Elements is the maintenance of the normal regularity of generation and restraint among the Elements(Zhanwen Liu, 2009).

\section{Reflections of the Restraining Circle in EFL (TB 》L 》 T 》TM》LS》TB)}

TB restraining L: A typical illustration to prove the concept of "textbooks restraining learners" lies in the fact that the comprehensible input (TB) can not "get in " (see Krashen,1982, pp.9-32) if learners (L) has high affective filter as they are "put on the defensive"(Stevick 1989). It is normally TB restraining L. However, if L are motivated, or have self-confidence and good self-image, or his level of anxiety is low, the comprehensible input can have its effect on acquisition. This is known as "L counter-restraining TE". In language learning, counter restraint is a positive phenomenon, indicating student' role being reversed. Students need to be engaged with the content of a text, not just its language (Jeremy Harmer,1998).

L restraining T: Alan C. Mcleam (2012) stresses that the need for all the teachers to consider learners as whole and integrated human beings and respond to them as such. Teachers should see English as a means of education, relating closely to the development of the learner's cognitive ability, rather than as simply the inculcation of a specific series of linguistic skills. Alan C. Mcleam thinks the need for learner-centered teaching is destroying the teacher.

The unique needs and different motivation of each student has restrained the teaching process of teacher's same administering technique in a whole class; The desire of developing an internalized thirst of knowledge and experience is conflicting with such extrinsic motivation as tests and exams in which schools all too often teach students to play the "game" of pleasing teachers and authorities. So in essence learners restrain teacher's innovative behavior (H. Douglas Brown, 1994, p.40).

T restraining TM: As Earth dams, muddies, or absorbs Water, the teacher should update his or her teaching methods so as to nourish the learners' growth (TM generating L). However, the concept of the venerable old master teacher is difficult to sustain in an educational context of new methodologies and new syllabuses (Sudsuang yutdhana, 2005), where the raw recruit from a College of Education may be better informed than the practicing teacher, as Wallace (1991, p.6) points out. Moreover, the teacher in traditional educational institutions is that of master controller, always in charge of every moment in the classroom. Nevertheless, some control on teacher's part is actually an important element of successfully carrying out interactive techniques(Wallace,1991), with which master controllers determine what teaching methods they will use, the Grammar Translation Method, the Direct Method, the Audio-lingual Method, TPR, the Natural Approach, and Communicative Language teaching.

TM restraining LS: Pintrich, P.R. \& DeGroot, E (1990) advocates that classroom interference is an effective mean to train learning strategies. That is an evidence reflecting the effect of WATER restraining FIRE, which research has become one of the most hopeful areas of research indirectly aimed at overcoming demotivation. Oxford Rebecca (1993) provides the most comprehensive taxonomy of learning strategies currently available, which are divided into what has come to be known as direct or cognitive strategies (Rebecca Oxford,1990), which learners apply directly to the language itself, and indirect or meta-cognitive strategies in which learners manage or control their own learning process (H. Douglas Brown, 1994, p.200). Teaching methods will perhaps be specifically geared toward building strategies competence. In Rebecca Oxford's (1993) Language Learning Strategies: What Every Teacher Ought to Know, the author recommends many different techniques, like encouraging risk-taking, promoting cooperative learning, encouraging them to use right-brain process and getting students to make lists of their common errors and to work on them on their own. Ernesto Macaro thinks that learning strategy training like function word strategy and keyword strategy should be taught to make them more responsible for their own learning (Ernesto Macaro, 2008, p.63). One of the best of teaching methods to get learners intrinsically involved in their language learning is to offer them the opportunity to develop their own set strategies for success. (H. Douglas Brown, 1994, p.80). Teachers have to care more about their students' learning than they do about their own teaching (Jeremy Harmer,2000). Therefore, teaching methods is controlling larning strategies. That's why there 
is a statement that the key to creating language classroom is the initiation of interaction by the teacher. One of the best ways to develop your role as an initiator and sustainer of interaction is to develop a repertoire of questioning strategies: knowledge questions, comprehension questions, application questions, inference questions: analysis questions, synthesis questions, evaluation (H. Douglas Brown, 1994, p.166).

LS restraining TB: Learners are hopeless when encountering textbooks with different features of genres, so their different learning strategies control the process of comprehending the text. For example, taking risk to ask question and make further exploration into the context determine them to go haw far in comprehension of the input. Learners tend to struggle in controlling the limited vocabulary and grammatical patterns within a textbook. So some learning strategies such as skimming, scanning, semantic mapping, and guessing can be encouraged to use to conquer the comprehension of the text. A few content-centered ESL textbooks are now appearing in which the content itself is the study and utilization of learning strategies(H. Douglas Brown, 1994, p.209).

\section{The Revelation of the Perceptible Five Teaching Elements}

The most revelation of the perceptible five Teaching Elements in EFL can help teachers to build the awareness of Whole Language Teaching. One of the most popular terms currently sweeping through EFL profession, "whole language" has been so widely and divergently interpreted that it unfortunately is on the verge of losing the impact that it once had(see Rigg, Pat. 1991). Rigg provides a great deal of research and background information on whole language education. The information in this article extends well beyond the whole language approach, extending into five aspects of teaching methods, learning strategies, learners, teacher, teaching materials or textbook). Whole language is a label that has been used to describe:

A. Cooperative and participatory learning (functioning as learning methods)

B. Focus on the community of learners (functioning as learners)

C. Focus on the social nature of language and use authentic, natural and meaning-centered language (functioning as teaching material or textbook)

D. Holistic assessment techniques in testing (functioning as teaching methods)

E. Sense of Integration of the "four skills" (functioning as teacher)

The above five factors are involved in an integration of two human body's developments, teacher and learners' growth, along with the learning strategies and teaching methods. As Shu Dingfang (2011, pp.5-6) pointed out that the classroom teaching has five functions: to develop students' interest in learning ( L-WOOD); to impart language knowledge in a systematic way (T-EARTH ); to enrich language learning environment and resources (TB-METAL); to provide guidance of foreign language learning methods and strategies (LS-FIRE); to provide opportunities for students to demonstrate learning outcomes (TM-WATER). From the perspective of five elements, the classroom teaching might function in harmonious way, if w teachers find the related solutions based on their interralationship.

As for solution to deal with some disordered teaching practice, Hu Wenzhong (1984) once said that learners (wood) often have psychological or emotional obstacles, so much that language input cannot reach the part of brain where language can be absorbed. Then the solution would be through various ways that vivid interesting language material (metal) can be selected, learners can be "submerged" in authentic language for "swimming in ocean"(fire), learners can be freed from the cumbersome of analysis of dull syntax explanation(water), which need the supports from well-qualified teacher (earth), the right learning materials (metal), and practical learning strategies (water), and so on.

\section{SUMMARY}

The purpose of this article is to apply the Chinese systematic way of thinking in ancient Chinese, Wuxing (the Five Elements), into EFL, even though they do not exactly follow the principle of the generating (productive effect), or the restraining (destructive effect) cycles. They really count in guiding treatment of some disorder of educational phenomena.

When teaching a language, the teacher also teach a complex system of cultural customs, values, and ways of thinking, feeling, and acting in learners' growth, their learning strategies and teacher's development. Based on the perspective of Five Teaching Elements, we might consider the dynamic balance among every element and find the right ways to deal with the troubling teaching issues, the ones addressed as "complementary pairs generating, and opposite pairs restraining". Anyway, this research opens up new paths for dynamic and effective classroom teaching in EFL field, even though some hypothetical classifications of elements and relationships could be valuable for future empirical tests and the classification form for attributes of Yin and Yang, as well as Five Teaching Elements might arise to dispute.

\section{ACKNOWLEDGEMENT}

This research was financially supported by the Provincal Philosophy and Social Science Foundation of Jiangsu, China (Grant NO. 2013SJB740032) and the Fundamental Research Funds for Soochow University (Grant NO. 5731501213).

\section{REFERENCES}

[1] Adrian S. Palmer.(1970). Teaching Communication. Language Learning, 6. 35-36. 
[2] Alan C. Mcleam.(2012). Destroying the Teacher: The Need for Learner-Centered Teaching. English Teaching Forum, 1. 36-38.

[3] Anna Craft. (2002). Creativity and Early Years Education: A Lifewide Foundation. New York: Continuum International Publishing Group Ltd.,195-196.

[4] Anthony, E.M. (1963). Approach, Method, Technique. English Language Teaching 17. 63-67.

[5] Ernesto Macaro. (2008). Teaching and learning a second language: A Guide to recent Research and its Application. Beijing: World Publication Company, 280-304.

[6] H. Douglas Brown. (1994). Teaching by Principles: An Interactive Approach to Language Pedagogy. Prentice Hall Regents, 190-213.

[7] Hu Wenzhong.(1984). Introduction to language acquisition and foreign language teaching - Stephen D. Krashen' principles and vision on English teaching. Foreign Language Studies, 1, 1-7.

[8] Atkin J. Myron. (1967). Research styles in science education, Journal of Research in Science Teaching, 12, 338-345.

[9] Jeremy Harmer. (2000). How to Teach English. Beijing: Foreign Language Teaching and Research Press.

[10] Krashen, S. (1982). Principle and Practice in Second Language Acquisition. Oxford: Pergamon Press.

[11] Liu Chang Lin. (1990). Chinese Systems Thinking. Beijing: China Social Science Press (in Chinese).

[12] Mattarima, Karim \& Hamdan, Abdul Rahim. (2011). Understanding Students: Learning Strategies as an Input Context to Design English Classroom Activities, International Journal of Psychological Studies.35-37.

[13] Neville Grant. (1987). Making the Most of Your Textbook. London: Longman Group Ltd.

[14] Rebecca L. Oxford. (1993). Language Learning Strategies: What Every Teacher Should Know. TESOL Quarterly, Volume 27, Issue 1,121-122.

[15] Pintrich, P.R.\& DeGroot, E. (1990). Motivational and self-regulated learning components of classroom academic performance. Journal of Educational Psychology, 82, 33-40.

[16] Rigg, Pat. (1991). Whole language in TESOL. TESOL Quarterly 25 (3), 521-542.

[17] Richard Jack \& Theodore Rodger. (1986). Approaches and Methods in Language Teaching. New York: Cambridge University Press.

[18] Chang Shih-Chuan. (2011). A Contrastive Study of Grammar Translation Method and Communicative Approach in Teaching English Grammar. English Language Teaching, 2, 13-21.

[19] Shu Dingfang. (2011). On the functions and objectives of the foreign language classroom teaching. Foreign language and foreign language teaching, 1, 5-6.

[20] Stevick, Earl.W. (1989). Success with Foreign Language.Virginia: Prentice Hall International.

[21] Sudsuang Yutdhana, (2005), The Development of a Teacher-training for Model in Using the Internet for Teaching English as a Foreign Language, http://www.Wtt.Org.Uk/Index.Html (accessed 19/9/2013).

[22] Wang Ningchuan \& Zou Yuze. (2011). Yin-Yang Theory and Globalization. Studies in Sociology of Science, 2, 36-38.

[23] Wang Aiping. (2012). Five Elements. http://www.five-element-theory.com (accessed 18/8/2013).

[24] Wallace, M.J. (1991). Traing Foreign Language Teachers: A Reflective approach. Cambridge: Cambridge University Press.

[25] Xia Songlin. (1989). Images of Heaven and Man: An Introduction to the History of Doctrines of Yinyang and Wuxing. Jinan, China: Shandong Literary Press (in Chinese).

[26] Liu Zhanwen. (2009), Formation of the Chinese Medicine Theoretical System and Its Philosophical Basis Essentials of Chinese Medicine. Essentials of Chinese Medicine(Liu Liang Edited). Springer London Ltd., 3-32.

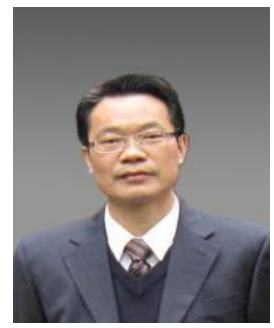

Bulei Zuo was born in Huai'an, China in 1965. He received his Master's degree in linguistics from Northeast Normal University, China in 2005. He is currently an associate professor in the School of Foreign Languages, Soochow University, Suzhou, China. His research interests include applied linguistics and English education. 